\title{
Capacitación docente: Pilar para la identificación y gestión de la violencia escolar
}

\section{Teacher training: Pillar for the identification and bullying management}

\begin{abstract}
Ramiro Andrés Andino Jaramillo es docente de la Unidad Educativa Nicolás Gómez Tobar (Ecuador) (raandinoj@gmail.com) (https://orcid.org/0000-0001-8547-8780)
\end{abstract}

Recibido: 2017-04-17 / Revisado: 2017-08-07 / Aceptado: 2017-11-01 / Publicado: 2018-01-01

\section{Resumen}

El presente trabajo se enfoca en explicar la mejora del proceso de identificación y gestión de la violencia escolar en los docentes de Educación General Básica de la Unidad Educativa "Nicolás Gómez Tobar", de la ciudad de Santo Domingo, período académico 2016-2017. La propuesta aplicada fue un módulo de capacitación que describe tipos de violencia escolar y modelos de gestión pacífica de conflictos entre estudiantes, que se pueden aplicar en el contexto educativo de la institución, ya que es necesario fortalecer la formación docente en gestión pacífica de casos de violencia escolar. La metodología del estudio se basó en la investigación explicativa y en un diseño cuasiexperimental, con la aplicación de un pretest, una intervención y un postest a una muestra de 22 docentes que imparten clases desde inicial hasta décimo grado en la institución. Las técnicas de investigación utilizadas fueron la encuesta y el análisis estadístico. Los resultados obtenidos con el estudio muestran que la capacitación mejora la identificación de casos de violencia escolar, de la misma manera, se plantea la posibilidad de aplicar uno o varios modelos de resolución pacífica de conflictos. En las conclusiones, se explica la importancia de la capacitación como parte del perfeccionamiento de la carrera docente en identificación y gestión de la violencia escolar, además se sintetizan los beneficios de concientizar a los docentes sobre este problema habitual en el contexto escolar.

Descriptores: Acoso escolar, capacitación, educación, docente, institución, violencia.

\begin{abstract}
This paper is focused on explaining the improving of the process of identification and management of school violence by teachers of primary education in the "Nicolás Gómez Tobar" school in Santo Domingo city during the period of 2016-2017. The applied proposal consisted of a training module which describes different types of school violence as well as models of pacific conflict management among students, which can
\end{abstract}

Forma sugerida de citar: Andino Jaramillo, R. A. (2018). Capacitación docente: Pilar para la identificación y gestión de la violencia escolar. Alteridad, 13(1), 108-119. https://doi.org/10.17163/alt.v13n1.2018.08. 
be applied in the educational context of the institution, ever since strengthen the pacific violence management is a real teacher's need. The methodology of this study was based on an explanatory research and quasi experimental design. With the application of a pretest, intervention and a posttest to a sample made up of twenty-two teachers, who teach from the initial to tenth grade. The applied research techniques were the survey and the statistical analysis. The results obtained through the research effectively show that training

\section{Introducción}

La violencia escolar es un problema cotidiano dentro de las aulas de clase, ya que se presenta de diferentes formas $y$, con una serie de víctimas y agresores diferentes. La Organización de las Naciones Unidas (ONU) considera a la violencia escolar como cualquier acto de agresión física o psicológica, lesiones, explotación, negligencia, omisión de denuncia que ocurre entre estudiantes, docentes y estudiantes, autoridades y estudiantes y contra la propiedad. Estos actos pueden ocurrir tanto dentro como fuera de la escuela, en la comunidad o barrio que está circundante con la institución, por redes sociales o cualquier tecnología de la información y la comunicación (Ministerio de Educación de Perú, 2014). Durante clases y fuera de ellas, los estudiantes experimentan una serie de conflictos, porque la institución educativa es un espacio social en el que conviven cientos de individuos con diferencias de todo tipo. Es inevitable considerar a la violencia escolar como un problema cotidiano de las instituciones educativas, ya que según López (2014) la violencia es una forma de resolver conflictos entre estudiantes, convirtiéndose así, en parte de la cotidianidad y convivencia de la comunidad educativa.

La violencia y las agresiones entre estudiantes se presentan de varias maneras en las instituciones educativas, forman parten de la cotidianidad de las aulas, por lo tanto, se debe considerar que la violencia se evidenciará en algún momento o situación; durante clases, improves school violence cases identification and also the possibility of applying one or several pacific conflict resolution models. The importance of teacher training on the violence identification and conflict management as part of the improvement of the teaching career is explained in the conclusions, as well as the benefits of making teachers aware of this habitual issue in the school context.

Keywords: Bullying, training, education, teacher, organization, violence.

recesos o en actividades escolares. Con respecto a lo mencionado, la Organización Mundial de la Salud (OMS) plantea que la violencia es el uso deliberado de la fuerza por medio de agresiones o la dominación, ya sea en forma de amenaza o hecho consumado, contra uno mismo, otras personas, un grupo o una comunidad. Este uso deliberado de fuerza por medio de agresiones, pueden causar muerte, lesiones, trastornos psicológico o de desarrollo madurativo (Piñero et al., 2014).

El problema de la violencia escolar se encarna en cada instancia de las instituciones educativas, por tanto es ineludible que no se generen conflictos en un espacio en el que se encuentran una diversidad de estudiantes con diferencias culturales y sociales. De esta manera, Piñero et al. (2014) en concordancia con la Organización Mundial de la Salud (OMS), considera que en la actualidad el problema de la violencia se reconoce como un problema social y de salud que produce un impacto nocivo en las condiciones de vida y estabilidad emocional de las personas, ya que la violencia se puede convertir en un factor generador de enfermedades a corto, medio o largo plazo, incluso provocar enfermedades crónicas.

Los primeros focos de atención y estudios científicos relacionados a la violencia escolar surgieron en los años 70, pero a partir de los años 80 en adelante se empezó a realizar investigaciones centradas en determinar los porcentajes de violencia que existían en las instituciones educativas y cómo afectaba a los estudiantes. En las pri- 
meras investigaciones sobre violencia escolar se centraba la atención sobre el individuo, es decir, el alumno y los efectos negativos que producen su contacto con algún tipo de violencia.

Esta perspectiva investigativa sobre el individuo tiene su origen en la gran influencia de las investigaciones realizadas por autores nórdicos que se centraban en factores o causas a nivel psicológico o familiar, enfocándose hacia aspectos netamente sociales y culturales (Colombo, 2011). A partir de esto, se plantearon algunas teorías que explicaban los efectos negativos de la violencia sobre las personas. La teoría del desarrollo por ejemplo, explicaba que los infantes que crecen en ambientes violentos, se adaptan a estos y desarrollan comportamientos agresivos que afectan su desarrollo emocional, social y psicológico (Rosser, Martínez \& Villegas, 2015).

Otro planteamiento fue la teoría del trauma que hacía énfasis en los cambios fisiológicos, el estrés y el desarrollo cerebral producto de la violencia experimentada por el individuo y cómo esto afectaba en gran medida el lenguaje, la memoria y la concentración. Las dos teorías antes mencionadas se centraban en contextos sociales fuera del hogar, por esa razón, se planteó la teoría de los sistemas familiares que consideran a la violencia escolar como resultado de la falta de apoyo que tenían los padres con respecto a infantes o adolescentes que eran agresivos dentro de las instituciones educativas.

Los resultados sobre violencia escolar en todos los países tienen porcentajes relativamente similares, en este sentido Cabezas \& Monge (2013) consideran que las conductas agresivas en las aulas de clase, se valoran alrededor de un $15 \%$, no obstante este porcentaje es variable de acuerdo a los estudios que se han realizado en diferentes contextos escolares, en otras palabras, la violencia dentro de las aulas de clase es una realidad presente en las instituciones educativas, aunque no sea evidente o se encubra, por esta razón, en América Latina y el Caribe se estima que entre un 50\% y $70 \%$ de estudiantes han sido testigos o víctimas de algún tipo de violencia, corroborando lo expuesto por el Ministerio de Educación del Ecuador (2014), el cual plantea que la violencia entre estudiantes se incrementa cada vez más en la región.

En 2004 se realizó una investigación científica a nivel nacional sobre violencia escolar en el Ecuador, a través del INNFA (Instituto Nacional de la Niñez y la Familia) en colaboración con el DNI (Defensa de Niños y Niñas Internacional). Con una muestra de niños y adolescentes se reveló los siguientes datos: El 32\% de los niños, niñas y adolescentes que hay en el Ecuador son golpeados o insultados "a veces" por compañeros de clase, de estos, el 22,6\% son niños y niñas entre edades de 6 a 11 años y, el 20,1\% son adolescentes entre 15 a 17 años de edad (McBride, 2012).

Otro informe con datos similares al anterior fue realizado en 2010 por el Observatorio de la Niñez y Adolescencia, revelando que el 63\% de los estudiantes en el Ecuador molesta a otros por ser diferentes; el $74 \%$ insulta o se burla de un compañero de clase; y el $53 \%$ sufre robos dentro y fuera de la institución (El Universo, 2014 , p. 1). Estos resultados demuestran cierta tendencia sobre el incremento progresivo de la violencia en las aulas de clase, a pesar de todas las propuestas o proyectos que se han realizado, y que aún continúan. Ante la masificación de la violencia y agresiones en el contexto escolar, el Ministerio de Educación del Ecuador ha pedido que los estudiantes, docentes, autoridades, padres de familia y la comunidad denuncien inmediatamente cualquier caso de acoso escolar (El Universo, 2014).

Son varias las explicaciones que se han dado para entender la violencia y las agresiones entre individuos, pero se desestima el hecho de que el maltrato entre iguales tiene un gran impacto en el contexto escolar, esto involucra agresiones y abusos sistemáticos dentro la relaciones interpersonales que se establecen entre estudiantes (Haro \& García, 2014), por esta razón, la violencia y agresiones dentro de las aulas de clase se presentan indistintamente dentro de las instituciones educativas, en sí, es un problema que atañe a toda la comunidad educativa, los legisladores y 
ciudadanos en general. Este trabajo enfoca el problema desde el planteamiento de Blásco \& Orgilés (2014), quienes afirman que la agresividad es una conducta que se aprende por medio de la observación de conductas agresivas que otros generan, siendo esto el estímulo para actuar de forma similar o con mayor brusquedad.

Como se lo ha mencionado, la violencia dentro de las aulas de clase es parte cotidiana de la convivencia escolar. Según varias investigaciones, los estudiantes y los docentes establecen relaciones interpersonales que pueden en algún momento convertirse en conflictivas, por lo tanto, parece ser claro que la capacitación docente es de importancia para gestionar los problemas que se puedan dar durante clases, y en mayor razón, si se trata de conflictos y agresiones entre estudiantes. De acuerdo a Martínez et al. (2007), los docentes son conscientes de los problemas emocionales, sociales, familiares y económicos de los estudiantes, y al no sentir ayuda o tener la capacitación necesaria para resolver situaciones precarias de sus discentes se generan estados emocionales de angustia, tristeza o frustración, provocando que estos cuestionen su desempeño docente, llegando incluso al escepticismo frente a nuevas iniciativas o propuestas en contra de la violencia en el contexto escolar.

La capacitación es parte del desarrollo profesional de los docentes, por el motivo que permite mejorar el proceso de enseñanza y aprendizaje. De acuerdo a Torrego, Monge, Pedrajas \& Martínez (2015) la formación del profesorado se la debe entender como un desarrollo profesional que mejora y dinamiza los procesos de aprendizaje, que constantemente están variando, por lo tanto, las prácticas profesionales deben desarrollarse en la misma manera que estos procesos. $\mathrm{La}$ perspectiva de estos autores valora a la capacitación como un medio de desarrollo profesional de los docentes, que en un sentido positivo, permite establecer mejores procesos de gestión de problemas observados en las aulas.

Son puntuales las investigaciones que proponen a la formación docente como medio para gestionar la violencia dentro de las aulas de clase, pese a ser una acción que no es de reciente planteamiento, sino más bien, forman parte de la mejora de los procesos de enseñanza y aprendizaje, además de fortalecer una convivencia escolar alejada de los conflictos, la violencia y otros problemas sociales. Sobre la base de lo mencionado, Gibbons \& Rossí (2015) consideran que los docentes deben promover experiencias significativas y relevantes para los estudiantes que estén en alguna situación de riesgo (violencia, exclusión, drogas, vandalismo, prostitución, etc.), en razón de que, las instituciones educativas que no dedican la atención necesaria para fomentar la convivencia pacífica, el liderazgo, la confraternidad, la colaboración, la cooperación y el empoderamiento; pueden acrecentar la violencia, la exclusión, el abuso y los conflictos dentro y fuera de las aulas de clase.

Mejorar la gestión de la violencia y los conflictos dentro del aula de clase es parte del perfil profesional que requieren los docentes. Como lo sostienen algunas investigaciones, la capacitación sobre violencia escolar, permite que los docentes brinden un mejor apoyo a los estudiantes que estén experimentado algún problema. De acuerdo a esto, Gibbons \& Rossí (2015) sostienen que los estudiantes con el apoyo necesario, pueden tener un cambio de actitud que permita ayudarlos a analizar situaciones cotidianas de forma distinta, impulsando el cambio de creencias, y de esta manera, desestimar a la violencia como parte funcional de la convivencia en la institución.

La capacitación de docentes sobre violencia escolar tiene la finalidad de prevenir y evitar actos violentos entre estudiantes, pero también es una forma de plantear mejores soluciones ante conflictos. Gibbons \& Rossí (2015) consideran que los objetivos de los programas de capacitación sobre violencia escolar no solo se deberían enfocar en prevenir la violencia y conflictos entre estudiantes, sino también en enseñar prácticas pacíficas de resolución de conflictos y toma de decisiones participativas que permitan fortalecer el clima positivo del aula y la convivencia armónica de todos los agentes de la comunidad educativa. 
Las investigaciones que se han realizado con respecto a la capacitación de docentes sobre violencia escolar, se enfocan no solo en la prevención de la violencia, sino también en mejores formas de gestionar el aula, para que no se generen conflictos agresivos entre estudiantes. Sobre la base de esto, Gibbons \& Rossí (2015) plantean que la formación docente en liderazgo y mediación forma parte de las decisiones a nivel individual y grupal para prevenir la violencia. El planteamiento de estos autores se relaciona con el rol de líder que debe tomar el docente ante conflictos y violencia entre estudiantes, para que cuando se tomen decisiones tengan un impacto positivo a nivel individual (docente o estudiante) como grupal (docentes y estudiantes, padres de familia, autoridades), y así solucionar la problemática abordada.

Distintos estudios que se enfocan en la capacitación docente en temas de violencia escolar, plantean que es posible la identificación de los tipos de violencia que se observan en el aula de clase como una medida para plantear estrategias de prevención y resolución pacífica de estos problemas. Martínez et al. (2007) sostienen que la identificación de los factores que generan o inhiben la agresión, podrían suministrar los procedimientos y acciones para diseñar estrategias de prevención e intervención para orientar políticas públicas que reduzcan el impacto de la violencia escolar, la cual se manifiesta precozmente en los niños y niñas.

De acuerdo a las perspectivas descritas, la violencia se presenta de forma frecuente en las aulas de clase, por esta razón, se han propuesto proyectos que ayudan a tratar este problema, que es una cuestión de interés para los docentes, estudiantes, padres de familia y la comunidad (Marchant, 2009). Sobre la base de la problemática identificada, se diseñó un módulo de capacitación con la finalidad de mejorar el proceso de identificación y gestión de la violencia escolar en los docentes de Educación General Básica de la Unidad Educativa "Nicolás Gómez Tobar", de la ciudad de Santo Domingo, período académico 2016-2017. La estructura del módulo contem- pla antecedentes de la violencia escolar a nivel internacional y nacional para situarla como un problema latente en la institución. La primera unidad del módulo tiene relación con las generalidades de la violencia; describiendo su tipología como es: violencia física, violencia psicológica, violencia sexual, violencia económica y violencia patrimonial, de la misma manera, se explican las modalidades en que se presenta la violencia, como son: intrafamiliar, institucional, laboral y violencia en contra de la naturaleza.

La segunda unidad aborda la violencia escolar, sobre la cual, se detalla y analiza casos de exclusión, hostigamiento, coacciones, intimidación y bullying (verbal, físico, gesticular, cibernético). De la misma manera, se describe el abstenimiento de la denuncia y los agentes que propician la violencia dentro de las instituciones educativas. La tercera unidad del módulo de capacitación tiene relación con los métodos de resolución pacífica de la violencia escolar. En este apartado temático se analiza y describe el modelo de técnica asertiva, el método Pikas, el círculo de amigos, la dinámica de mediación y el modelo relacional integrado. En este sentido, el trabajo presentado contempla la importancia de mejorar el proceso de identificación y gestión de la violencia escolar, que es una necesidad prioritaria en la formación de los docentes, porque en ciertos casos, estos no tienen los conocimientos ni teóricos, ni metodológicos para gestionar conflictos o agresiones dentro del aula de clase (Vijayan, Chakravarthi, Philips, 2016).

\section{Material y métodos}

El proceso metodológico aplicado en la investigación es poco común, ya que trabajos con temáticas similares al que se propone en este manuscrito, se centran en estudios de caso y otros diseños metodológicos no experimentales. Desde esta perspectiva, la propuesta investigativa tomó un diseño cuasiexperimental que considera resultados antes, durante y después de la intervención, para determinar los alcances y las 
limitaciones de la investigación. Sobre la base de la metodología aplicada y fundamentada en el enfoque mixto (cualitativo y cuantitativo), el alcance de la investigación abarcó de manera más amplia el problema de la violencia escolar en la institución, incluso permitió una mayor generalización de los resultados.

Como se lo mencionó en el párrafo anterior, el proceso metodológico del estudio se fundamentó en un enfoque mixto, debido a que se utilizó el enfoque cualitativo para analizar la experiencia docente con respecto a la capacitación. Del mismo modo, se aplicó el enfoque cuantitativo para el análisis estadístico de la mejora en la identificación y gestión de la violencia escolar, una vez concluida la capacitación. El diseño cuasiexperimental, según Hernández, Fernández \& Baptista (2010) es útil para la medición de variables sociales, considerando principalmente que se escogen grupos previamente formados, es decir no se emplea ningún tipo de selección aleatoria. En investigación educativa, este tipo de diseño permite alcanzar resultados para tendencias generales, porque las variables de estudio pueden ser comparadas antes, durante $y$ después, en un tiempo determinado.

Para el presente trabajo, el diseño cuasiexperimental se aplicó mediante un pretest, una intervención y un postest para una muestra intencional de 22 docentes de Educación General Básica que imparten clases desde inicial hasta décimo grado en la institución. En el pretest, se aplicó una encuesta de 20 preguntas relacionadas a casos de violencia escolar y modelos de gestión pacífica de conflictos. A partir de la encuesta, se propuso el módulo de capacitación con tres unidades que detallan los tipos de violencia escolar y modelos de gestión pacífica de conflictos que se pueden contextualizar con la realidad de la comunidad educativa. La capacitación se realizó durante quince días y dos horas de participación diaria. La estrategia metodológica fue participativa; por lo tanto, se realizó debates y mesas de trabajo para el análisis de las unidades temáticas y el desarrollo grupal de los talleres del módulo. El postest consistió en volver a aplicar la encuesta tomada en el pretest, y de esta manera, comparar estadísticamente los resultados alcanzados para determinar si hubo una mejora en el proceso de identificación y gestión de la violencia escolar.

\section{Análisis y resultados}

A continuación se describen y analizan los resultados de las preguntas generales y específicas de la encuesta sobre identificación y gestión de violencia escolar, aplicada a 22 docentes de Educación General Básica de la Unidad Educativa "Nicolás Gómez Tobar".

Tabla 1. Aspectos generales de los docentes de la institución

\begin{tabular}{|l|l|}
\hline \multirow{2}{*}{ Formación profesional } & Tercer nivel $(86,36 \%)$ \\
\cline { 2 - 2 } Formación pedagógica & Cuarto nivel (13,64\%) \\
\hline Capacitación en resolución pacífica de la violencia escolar & $100 \%$ \\
\hline \multirow{2}{*}{ Frecuencia de capacitación } & $86,36 \%$ \\
\hline Disposición para capacitación & Hace un mes (4,55\%) \\
\cline { 2 - 2 } & Hace un año (22,73\%) \\
\cline { 2 - 2 } Frecuencia de observación de conflictos & Hace más de dos años (72,73\%) \\
\hline & $100 \%$ \\
\hline
\end{tabular}

Fuente: elaboración propia, datos obtenidos de la encuesta sobre identificación y gestión de la violencia escolar. 
De acuerdo con los resultados, los docentes de la institución tienen una formación profesional de tercer nivel $(86,36 \%)$ y cuarto nivel $(13,64 \%)$, de la misma manera, todos tienen una formación pedagógica; por lo tanto, son profesionales formados para ejercer la docencia (ver tabla 1). En contraste con los resultados de la formación profesional, el 86, 36\% de los docentes indica que ha sido capacitado para resolver pacíficamente la violencia escolar; sin embargo, parece ser que existe un escaso proceso de capacitación; por el motivo que, el mayor porcentaje de docentes $(72,73 \%)$ indica que hace más de dos años ha recibido una capacitación en resolución pacífica de la violencia escolar (ver tabla 1).

A pesar de que se evidencia una escasa frecuencia de capacitación a docente, todos los encuestados están dispuestos a capacitarse en temas relacionados a la gestión de la violencia escolar (ver tabla 1). En contraste con los resultados obtenidos, se muestra que el $86,37 \%$ de los docentes observa semanalmente conflictos o agresiones entre estudiantes, de la misma manera, el 9,09\% indica que mensualmente observa actos violentos entre estudiantes. A diferencia de los docentes que observan actos violentos entre estudiantes, el $4,55 \%$ de encuestados indica que no observa ninguna agresión entre estudiantes (ver tabla 1); por lo tanto, parece ser que existe la posibilidad que haya docentes que no tienen la capacidad de identificar tipos de violencia escolar o, por el contrario, no son conscientes del problema observado por los demás docentes.

Tabla 2. Identificación de tipos de violencia escolar

\begin{tabular}{|c|c|c|c|}
\hline Tipo de violencia escolar & $\begin{array}{c}\text { Pretest } \\
\text { (correcta) }\end{array}$ & $\begin{array}{c}\text { Postest } \\
\text { (correcta) }\end{array}$ & Mejora \\
\hline Coacciones & $9,09 \%$ & $95,24 \%$ & $86,15 \%$ \\
\hline Intimidación & $36,36 \%$ & $95,24 \%$ & $58,87 \%$ \\
\hline Hostigamiento & $18,18 \%$ & $95,24 \%$ & $77,06 \%$ \\
\hline Bullying gesticular & $31,82 \%$ & $100 \%$ & $68,18 \%$ \\
\hline Bloqueo social & $54,55 \%$ & $100 \%$ & $45,45 \%$ \\
\hline Bullying verbal & $77,27 \%$ & $100 \%$ & $22,73 \%$ \\
\hline Ciberbullying & $72,73 \%$ & $100 \%$ & $27,27 \%$ \\
\hline Promedio de mejoras & & & $55,10 \%$ \\
\hline
\end{tabular}

Fuente: elaboración propia, datos obtenidos de la encuesta sobre identificación y gestión de la violencia escolar.

En concordancia con los resultados del pretest, se muestra que los docentes identifican en menor porcentaje las coacciones $(9,09 \%)$, el hostigamiento $(18,18 \%)$, el bullying gesticular $(31,82 \%)$ y la intimidación $(36,36 \%)$; por el contrario, los tipos de violencia escolar que identifican de mejor manera son el bullying verbal $(77,27 \%)$, el ciberbullying $(72,73 \%)$ y el bloque social $(54,55 \%)$ (ver tabla 2$)$.

Luego de la aplicación de la capacitación a los docentes, los resultados del postest indican que los docentes mejoraron la identificación de las coacciones, la intimidación y el hostigamiento en un $94,24 \%$ respectivamente. De la misma manera, los resultados indican un $100 \%$ de identificación en el bullying gesticular, bloqueo social, bullying verbal y ciberbullying (ver tabla 2). Al realizar una comparación de los porcentajes de identificación de tipos de violencia escolar en el pretest y el postest, se obtiene un promedio mejora del 55,10\% (ver tabla 2), que indica un incremento sustancial en la diferenciación y tipificación de tipos de violencia escolar. 
Tabla 3. Identificación y utilización de modelos de gestión de violencia escolar

\begin{tabular}{|l|l|l|l|}
\hline \multicolumn{1}{|c|}{ Gestión pacífica de violencia escolar } & \multicolumn{1}{c|}{$\begin{array}{c}\text { Pretest } \\
\text { (correcta) }\end{array}$} & \multicolumn{1}{c|}{$\begin{array}{c}\text { Postest } \\
\text { (correcta) }\end{array}$} & \multicolumn{1}{c|}{ Mejora } \\
\hline Método Pikas & $13,64 \%$ & $85,71 \%$ & $82,08 \%$ \\
\hline Modelo relacional integrado & $22,73 \%$ & $95,24 \%$ & $72,51 \%$ \\
\hline Círculo de amigos & $13,64 \%$ & $76,19 \%$ & $62,55 \%$ \\
\hline Dinámica de Mediación & $54,55 \%$ & $95,24 \%$ & $40,69 \%$ \\
\hline Modelo de Técnica Asertiva & $40,91 \%$ & $76,19 \%$ & $35,28 \%$ \\
\hline Promedio de mejoras & & & $58,62 \%$ \\
\hline
\end{tabular}

Fuente: elaboración propia, datos obtenidos de la encuesta sobre identificación y gestión de la violencia escolar.

Con relación a los resultados del pretest, se demuestra que el método Pikas (13,64\%), el círculo de amigos $(13,64 \%)$ y el modelo relacional integrado $(22,73 \%)$ son los modelos de que los docentes menos identifican o utilizarían para resolver conflictos o agresiones entre estudiantes (ver tabla 3 ); por otro lado, la dinámica de mediación $(54,55 \%)$ y el modelo de técnica asertiva $(40,91 \%)$ en el pretest, son los modelos que identifican y utilizarían los docentes para gestionar la violencia escolar (ver tabla 3).

Una vez realizada la capacitación, los resultados indican que los docentes tienen una mejor capacidad para identificar y proponer la utilización de los modelos propuestos en la capacitación (mayor al 76\%, ver tabla 3). La comparación entre el pretest y el postest indica un promedio de mejora del $58,52 \%$ en la identificación y utilización de los modelos propuestos (ver tabla 3). Sobre la base de la comparación entre el pretest y postest y las mejoras por cada modelo, se propone al modelo relacional integrado $(95,25 \%)$ como la alternativa que la mayoría de docentes utilizaría para gestionar conflictos en el aula de clase (ver tabla 3 ).

\section{Discusión}

Sobre la base de los resultados, se demuestra que los docentes tienen formación de tercer y cuarto nivel en formación pedagógica, pero su capacitación en resolución pacífica de violencia escolar es poco frecuente o inexistente, provocando que posiblemente los docentes se conviertan sin proponérselo en cómplices de la violencia, como lo sostienen Cabezas \& Monge (2013). De la misma forma, los docentes conocen y observan actos violentos dentro y fuera del aula; por lo tanto, parece ser que sienten la responsabilidad y necesidad de hacer algo para mejorar esta problemática, aunque no sepan cómo hacerlo. Con base a la necesidad de capacitación identificada y el conocimiento del problema de la violencia escolar que tienen los docentes, parece ser necesaria la mejora de los procesos de perfeccionamiento docente en temas de identificación de la violencia escolar, ya que según Gómez (2013), posiblemente los docentes se conviertan en espectadores pasivos de los conflictos entre estudiantes, dando como resultado un incremento de la violencia dentro y fuera de las aulas de clase.

Aunque se evidencia una poca o inexistente capacitación en resolución pacífica de la violencia escolar, los docentes parecen estar sintiéndose corresponsables de este problema, ya que de acuerdo a Lorente, Ramos \& Pérez (2016), los docentes son conscientes de los problemas emocionales, sociales, familiares y económicos de los estudiantes. Desde esta perspectiva Rodríguez (2014) plantea la importancia de la capacitación como un medio para mejorar las competencias docentes en resolución pacífica de la violencia escolar, tomando en consideración que los docentes son conscientes de las virtudes, nece- 
sidades y problemas de sus estudiantes; por lo tanto, la capacitación sería una de las herramientas para que los docentes acepten nuevas propuestas, pero también que tengan la capacidad de generar propuestas que se ajusten al contexto educativo y al conflicto observado y evaluado en cada aula de clases.

Si los docentes no se concientizan sobre la violencia que se genera en el contexto escolar, tampoco pueden concienciar a los estudiantes sobre esta problemática, que según Gairín, Armegol \& Silva (2013) puede aumentar la apatía e insolidaridad con respecto al sufrimiento de las víctimas, aumentando el riesgo de que existan en el futuro nuevos protagonistas directos de la violencia. Atendiendo a este planteamiento, Castro \& Regattieri (2012) consideran necesario fortalecer la solidaridad y tolerancia recíproca para equilibrar la vida social que se da dentro y fuera del aula de clases, porque la violencia no solo afecta a la víctima o al agresor, sino también a quiénes la observan y no la denuncian por miedo o por indolencia del conflicto que se produce.

La disposición y responsabilidad de los docentes por capacitarse es parte del proceso de mejora y solución de la violencia escolar; en razón de que, Ruiz (2014) considera que los docentes deben promover experiencias significativas para los estudiantes, además Gibbons \& Rossí (2015) plantean que las escuelas que no dedican atención a mantener la unidad, el liderazgo, la renovación y la colaboración, pueden acrecentar la violencia, la exclusión y los conflictos entre estudiantes y docentes. De acuerdo con la explicación de los autores, resulta importante que cada docente sea un promotor de mecanismos para resolver pacíficamente conflictos y, sobre la base de propuestas cercanas a la realidad del contexto escolar, plantear situaciones significativas que sean de provecho para los estudiantes y una posible solución del problema de la violencia escolar.

Otro aspecto relevante de la disposición y responsabilidad de los docentes por mejorar los procesos educativos, lo consideran Gibbons \& Rossí (2015), los cuales sostiene que si los estu- diantes cuentan con el apoyo suficiente, pueden cambiar su actitud, además de ayudarlos a analizar de diferente manera las situaciones diarias a las que se enfrentan, que de una u otra manera, podría dar paso a un cambio de creencia sobre la violencia. Con base a la perspectiva planteada, parece ser que el módulo de capacitación aportó en la concienciación de los docentes sobre la violencia escolar que existe en la institución, ya que hubo mejoras en la identificación y propuesta de solución ante esta problemática; por lo tanto, se viene a demostrar que el conocimiento de los tipos de violencia escolar por parte de los docentes de la Unidad Educativa "Nicolás Gómez Tobar" incide positivamente en la identificación y el reconocimiento de conflictos o actos violentos dentro de las aulas de clase.

La concienciación de la violencia escolar por parte de los docentes, surge del conocimiento e identificación de su tipología en el contexto escolar, porque según Gibbons \& Rossí (2015) la capacitación sobre violencia escolar no solo se enfoca en prevenir este problema, sino también en enseñar mejores estrategias para la solución de conflictos y la toma de decisiones para mejorar la comunicación y relaciones interpersonales entre estudiantes y docentes. Efectivamente, de acuerdo con Martínez et al. (2007) la identificación de los factores que generan la violencia, podría proporcionar las pautas para mejorar, plantear y desarrollar estrategias de intervención y políticas públicas que se orienten a la lucha en contra de de la violencia dentro y fuera de las aulas de clase.

A partir de que los resultados muestran una responsabilidad de los docentes con respecto a la violencia, también hay una aceptación unánime de los mismos por capacitarse en resolución pacífica de la violencia escolar; por lo tanto, parece ser que el modelo relacional integrado con respecto a los otros métodos de resolución pacífica de la violencia escolar, tiene una mayor posibilidad de aplicación en la institución, ya que, los docentes y estudiantes están dispuestos a participar en la mejora de la situación. Aunque los resultados muestran una mayor posibilidad 
de aplicación del modelo relacional integrado, es necesario resaltar que el método Pikas, el círculo de amigos, el modelo de técnica asertiva y la dinámica de mediación son métodos que se pueden aplicar positivamente, de acuerdo al caso de violencia escolar que se identifique.

En respuesta a la predisposición de los docentes y al cambio de creencias de los estudiantes, se plantea el modelo relacional integrado como un método adecuado para el contexto educativo de la institución, porque traslada la resolución pacífica de la violencia escolar a todos los actores de la comunidad educativa, esto quiere decir que, desde la propia iniciativa o animados por los docentes y padres de familia, los estudiantes resuelven conflictos y agresiones por medio del diálogo pacífico y la conciencia sobre el impacto de la violencia dentro y fuera del aula de clase. De este modo, parece ser que existe una posibilidad latente de llevar a cabo este modelo de resolución pacífica de violencia escolar en la institución, pues los docentes y estudiantes han expuesto su interés en participar en la resolución pacífica de esta problemática que aún parece ser una muy radical para todos los actores de la comunidad educativa, encontrándose por otro lado, habituada en el contexto educativo.

En definitiva, la capacitación docente en temas relacionados a la identificación y gestión de la violencia escolar permite cambiar la perspectiva del problema; de modo que, da la posibilidad de proponer estrategias que coadyuven en la lucha de la violencia escolar. A partir de la capacitación de los docentes, se evidencia mediante los resultados una mejora en la identificación de tipos de violencia escolar, pero también parece ser que existe la posibilidad de ejecutar modelos participativos de gestión pacífica de la violencia escolar, como es el caso del modelo relacional integrado, el cual fue mayormente aplicado por los docentes luego de haber concluido con la capacitación. Con base a esta apertura positiva de los docentes, se puede resumir que la capacitación es una herramienta que a más de concientizar al docente sobre el problema de la violencia, le permite tomar un rol de líder con respecto a propuestas que ayuden a mitigar la violencia escolar en cada aula de clase.

\section{Conclusiones}

En términos generales, la capacitación de los docentes con respecto a la resolución pacífica de la violencia escolar es poco frecuente o inexistente, provocando que los conflictos y agresiones entre estudiantes sean poco identificados o controlados en la institución. Esta arista del problema determinada en el estudio se relaciona con la necesidad de perfeccionamiento de la carrera docente, ya que es claro que los docentes están dispuestos a capacitarse en resolución pacífica de conflictos y agresiones entre estudiantes; por lo tanto, existe la posibilidad de aplicar modelos integrales de participación de la comunidad educativa, tal es el caso del modelo relacional integrado, el método Pikas, modelo de técnica asertiva, el círculo de amigos y la dinámica de mediación. Estas posibles soluciones para el problema de la violencia escolar no surgen de manera espontánea, sino que son el resultado de un proceso de concienciación y formación del docente para expandir y proponer modelos que se contextualicen con la realidad escolar en la que se suscitan conflictos y agresiones entre estudiantes.

Con respecto a la aplicación del módulo de capacitación, se observó un cambio de actitud de los docentes, ya que en primer lugar, hubo una aceptación positiva de la capacitación, y en segundo lugar, los docentes identificaban específicamente y con mayores resultados los tipos de violencia escolar que se producía en sus aulas de clase, además, procedimentalmente proponían el modelo de gestión pacífica de violencia escolar que mejor se contextualizara con las características del conflicto identificado. Este cambio de creencias y procedimientos de los docentes es reflejo de la capacitación en identificación de tipos de violencia escolar y modelos de gestión pacífica de conflictos, que indudablemente 
forma parte del proceso de perfeccionamiento de la carrera docente.

Es claro que la capacitación sobre violencia escolar es un elemento necesario para desarrollar conciencia en los docentes sobre este problema cotidiano entre estudiantes. De acuerdo con lo planteado, es posible fomentar una cultura de prevención y tratamiento de conflictos mediante la capacitación a docentes en temas relacionados con la identificación de tipos de violencia escolar y los métodos para gestionarla de manera pacífica. En este sentido, existe la posibilidad de mejorar esta problemática, porque a partir de la capacitación los docentes poseen los conocimientos y procedimientos necesarios para gestionar la violencia escolar que observen dentro de sus aulas de clase.

En definitiva, la oportunidad que se establece con respecto a la capacitación docente, permite desde cualquier perspectiva un aspecto positivo en la mejora del proceso educativo que se da en la institución educativa. La formación docente es un proceso inacabado que requiere de un perfeccionamiento progresivo mediante un proceso de capacitación que se adecue a las necesidades del profesional de la educación y, de acuerdo a los resultados de mejora obtenidos con el estudio, la capacitación sobre identificación y resolución pacífica de la violencia escolar es un elemento trascendental en la lucha de habituación de la violencia, en una sociedad cada vez más inconsciente de problemas fundamentales que coartan la convivencia, la interculturalidad, el cambio y la paz. En definitiva, el módulo de capacitación sobre identificación y gestión de la violencia escolar resultó ser efectivo en el contexto escolar de la institución y se adecuó a las necesidades del profesorado que labora en cada uno de los grados de Educación General Básica.

\section{Referencias bibliográficas}

Blasco, M. \& Orgilés, M. (2014). Agresividad en menores de 18 años jugadores de fútbol: Diferencias en función del sexo y la edad y en comparación con los jugadores de baloncesto.
Cuadernos de Psicología del Deporte, 14(2), 21-26. Recuperado de https://goo.gl/r1jPyd

Cabezas, H. \& Monge, M. (2013). Violencia escolar, un problema que aumenta en la escuela primaria costarricense. Actividades Investigativas en Educación, 13(2), 1-20. Recuperado de https://goo.gl/LmrCre

Castro, J. \& Regattieri, M. (2012). Interacción Escuela familia. Brasilia: UNESCO. Recuperado de https://goo.gl/hcX7m3

Colombo, G. (2011). Violencia escolar y convivencia escolar: Descubriendo estrategias en la vida cotidiana escolar. Revista Argentina de Psicología, 8-9(15-16), 81-104. Recuperado de https://goo.gl/nkysJR

El Universo (2014). El acoso escolar se torna más violento en las aulas de Ecuador. Recuperado de https://goo.gl/7zA4DU

Gairín, J., Armengol, C., \& Silva, B. (2013). El 'bullying' escolar. Consideraciones organizativas y estrategias para la intervención. Educación XXI, 16(1), 17-38. Recuperado de https://goo.gl/prHYZi

Gibbons, M. \& Rossí, M. (2015). Evaluación de Impacto de un Programa de Inclusión Social y Prevención de Violencia Estudiantil. Washington: BID. Recuperado de https://goo.gl/qKbHq6

Gómez, A. (2013). Bullying: El poder de la violencia. Una perspectiva cualitativa sobre acosadores y víctimas en escuelas primarias de Colima.Revista Mexicana de Investigación Educativa, 18 (58), 839-870. Recuperado de https://goo.gl/tWKT5s

Haro, I. \& García, B. (2014). Variables emocionales y sociomorales asociadas con el tipo de rol que asumen los alumnos y alumnas en el maltrato entre iguale. Apuntes de Psicología, 32(1), 15-23. Recuperado de https://goo.gl/Zg6cAV

Hernández, R., Fernández, C. \& Baptista, M. (2010). Metodología de la investigación. 5ta. ed. México: Mcgraw-Hill.

López, V. (2014). Convivencia escolar. Santiago: UNESCO. Recuperado de https://goo.gl/U2ZZ7M

Lorente, L., Ramos, G., \& Pérez, A. (2016). Las prácticas docentes y el desarrollo de las competencias emocionales en estudiantes de educación primaria. Aula de Encuentro, 18 (1), 129-154. Recuperado de https://goo.gl/guJolG 
Marchant, C. (2009). Sobre reformas, integración inclusión y exclusión educativa. Reflexiones a partir de la experiencia española. Revista Latinoamericana de Educación Inclusiva, 3(2), 15-25.

Martínez, J., Muñoz, L., Duque, A., Castrillón, D., Rojas, C. \& Tovar, J. (2007). Percepciones y creencias de los docentes de algunas escuelas públicas de Pereira sobre el comportamiento agresivo de los niños. Revista Investigaciones Andina, 9(14), 5-25. Recuperado de https://goo.gl/JEKgC1

McBride, C. (2012). Bullying en el Ecuador. Quito: USFQ.

Ministerio de Educación de Perú (2014). Paz escolar, estrategia nacional contra la violencia escolar. Lima: MINEDU. Recuperado de https://goo.gl/qSjQQ3

Piñero, E., Arense, J., López, J. \& Torres, A. (2014). Incidencia de la violencia y victimización escolar en estudiantes de educación secundaria obligatoria en la Región de Murcia. Revista de Investigación Educativa, 32(1), 223-241. Recuperado de https://goo.gl/RMPjhy

Rodríguez, D. (2014). La mediación escolar: claves de interpretación desde una mirada politi- co-educativa. Tandil: NEES. Recuperado de https://goo.gl/Vq9rFy

Rosser, A., Martínez. R. \& Villegas, E. (2015). La exposición a violencia de género y su repercusión en la adaptación escolar de los menores. Bordón, 67(2), 117-129. Recuperado de https://goo.gl/DD5oJV

Ruiz, M. (2014). Liderazgo y responsabilidad educativa: el necesario liderazgo de directores y profesores en la educación. Revista Fuentes, 1 (14), 85-104. Recuperado de https://goo.gl/mbvR3A

Torrego, J., Monge, C., Pedrajas, M. \& Martínez, C. (2015). Formación del profesorado en aprendizaje cooperativo y alumnos con altas capacidades: un enfoque inclusivo. Revista Latinoamericana de Educación Inclusiva, 9 (2), 91-110. Recuperado de https:/goo.gl/9jc2DT

Vijayan, P., Chakravarthi, S. \& Philips, J. (2016). The Role of Teachers' Behaviour and Strategies in Managing a Classroom Environment. International Journal of Social Science and Humanity, 6(3), 208-215. Recuperado de https://goo.gl/yYpcxh 